\title{
Power spectra of infragravity waves in a deep ocean
}

\author{
Oleg A. Godin,, ${ }^{1,2}$ Nikolay A. Zabotin, ${ }^{1,3}$ Anne F. Sheehan, ${ }^{1,4}$ Zhaohui Yang, ${ }^{4}$ and \\ John A. Collins ${ }^{5}$ \\ Received 5 February 2013; revised 24 March 2013; accepted 25 March 2013; published 29 May 2013.
}

[1] Infragravity waves (IGWs) play an important role in coupling wave processes in the ocean, ice shelves, atmosphere, and the solid Earth. Due to the paucity of experimental data, little quantitative information is available about power spectra of IGWs away from the shore. Here we use continuous, yearlong records of pressure at 28 locations on the seafloor off New Zealand's South Island to investigate spectral and spatial distribution of IGW energy. Dimensional analysis of diffuse IGW fields reveals universal properties of the power spectra observed at different water depths and leads to a simple, predictive model of the IGW spectra. While sources of IGWs off New Zealand are found to have a flat power spectrum, the IGW energy density has a pronounced dependence on frequency and local water depth as a result of the interaction of the waves with varying bathymetry. Citation: Godin, O. A., N. A. Zabotin, A. F. Sheehan, Z. Yang, and J. A. Collins (2013), Power spectra of infragravity waves in a deep ocean, Geophys. Res. Lett., 40, 2159-2165, doi:10.1002/grl.50418.

\section{Introduction}

[2] Infragravity waves (IGWs) are surface gravity waves in the ocean with periods longer than the longest periods $(\sim 30 \mathrm{~s})$ of wind-generated waves. IGWs propagate transoceanic distances with very little attenuation in deep water and, because of their long wavelengths (from $\sim 1 \mathrm{~km}$ to hundreds of kilometers), provide a mechanism for coupling wave processes in the ocean, ice shelves, the atmosphere, and the solid Earth [Crawford et al., 1998; Rhie and Romanowicz, 2004; Webb, 2007; Uchiyama and McWilliams, 2008; Bromirski et al., 2010; Bromirski and Stephen, 2012]. Recent theoretical results [Godin and Fuks, 2012] suggest that IGWs with frequencies below $\sim 4 \mathrm{mHz}$ may be an efficient conduit of mechanical energy from the ocean into the upper atmosphere.

\footnotetext{
${ }^{1}$ Cooperative Institute for Research in Environmental Sciences, University of Colorado, Boulder, Colorado, USA.

${ }^{2}$ National Oceanic and Atmospheric Administration/Earth System Research Laboratory, Boulder, Colorado, USA.

${ }^{3}$ Department of Electrical and Computer Engineering, University of Colorado, Boulder, Colorado, USA.

${ }^{4}$ Department of Geological Sciences, University of Colorado, Boulder, Colorado, USA.

${ }^{5}$ Woods Hole Oceanographic Institution, Woods Hole, Massachusetts, USA.

Corresponding author: O. A. Godin, Cooperative Institute for Research in Environmental Sciences, University of Colorado, Boulder, CO, USA. (oleg.godin@noaa.gov)

(C)2013. American Geophysical Union. All Rights Reserved. 0094-8276/13/10.1002/grl.50418
}

[3] Most field observations of IGWs have been made in relatively shallow water on continental shelves [Munk, 1949; Herbers et al., 1995; Sheremet et al., 2002]. Deepwater IGWs [Snodgrass et al., 1966; Filloux, 1983; Webb et al., 1991] are among the least studied waves in the ocean; and their temporal and spatial variability remains poorly understood [Dolenc et al., 2005; Uchiyama and McWilliams, 2008]. Moreover, little quantitative information is available about IGW power spectra [Webb and Crawford, 2010]. This is primarily due to IGW amplitudes on the ocean surface being much smaller than the amplitudes of wind waves and swell, and the resulting need for precision measurements from stable platforms. Because of hydrodynamic filtering (attenuation of surface waves with depths on the scale of their wavelength), the relation of amplitudes of short- and long-period surface gravity waves is reversed at large depths. Therefore, measurements with seafloor sensors [Filloux, 1983; Webb et al., 1991; Dolenc et al., 2005, 2008; Sugioka et al., 2010] prove to be the main source of quantitative information about deepwater IGWs.

[4] While the properties of IGWs near the seafloor are of direct relevance to seismic applications, the oceanographic significance of IGWs and their possible coupling to the atmosphere are better characterized by spectra of either depth-integrated wave energy or ocean surface elevations due to IGWs. Such a characterization of IGWs would be consistent with the accepted spectral description of surface gravity waves in the other frequency bands [Komen et al., 1984]. Much like the modulation of spectra of wind waves by horizontally inhomogeneous surface currents [Godin and Irisov, 2003], spatial variations in bathymetry affect propagation of IGWs and are expected to result in systematic spatial variations of the measured IGW spectra.

[5] This paper presents the first analytical model of the spectral and spatial energy distribution of IGW energy in a deep ocean with variable bathymetry. The model is derived from long-term observations of IGWs off New Zealand and the basic physics of wave fields generated by random sources in an inhomogeneous medium.

\section{Observations}

[6] Extensive data on infragravity waves over varying bathymetry away from the shore were obtained in the Marine Observations of Anisotropy Near Aotearoa (MOANA) Seismic Experiment. MOANA was an ocean-bottom seismic experiment deployed from January 2009 to February 2010 offshore the South Island of New Zealand (Figure 1) [Yang et al., 2012]. The experiment was designed for studies of tectonic plate boundary processes through mantle seismic imaging. Thirty stations were deployed at approximately $100 \mathrm{~km}$ spacing and at water depths from 550 to $4680 \mathrm{~m}$. In addition to a broadband ocean bottom seismometer (OBS), 


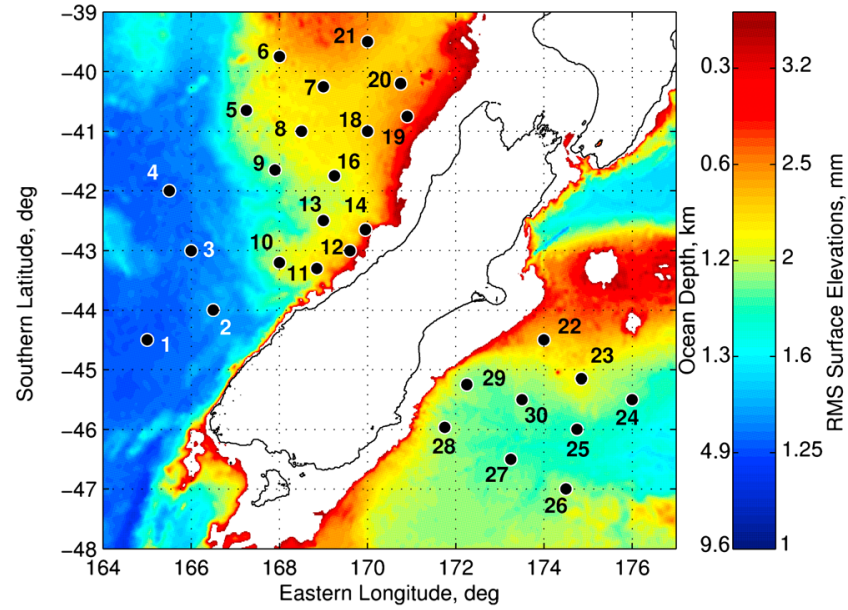

Figure 1. Location of seafloor pressure sensors in the MOANA experiment (numbered circles). Color shows bathymetry and RMS elevations of the ocean surface due to background infragravity waves in the frequency range 0.5 $30 \mathrm{mHz}$ as predicted by the power spectrum model developed in this paper. Areas with water depth less than $200 \mathrm{~m}$, where the model is not applicable, are shown in white.

each station was equipped with a Cox-Webb deepwater differential pressure gauge (DPG). DPGs measure variations of pressure on the seafloor with frequencies above $\sim 1 \mathrm{mHz}$ [Cox et al., 1984; Webb et al., 1991]. Data were recorded continuously at 50 samples per second and stored in daylong data segments. Yearlong pressure records were successfully retrieved from 28 stations.

[7] Pressure sensors were not individually calibrated and were instead characterized by a nominal (average) transfer function [Willoughby et al., 1993]
[9] Each daylong recording produced three overlapping data segments, each of $T_{M 2}$ duration. The segments were chosen at the beginning, middle, and end of the recording, respectively. We are interested in ambient (background) IGW field as opposed to IGWs associated with rare, strong, transient events such as earthquakes and tsunamis. To separate background IGWs, the data segments were automatically screened for the presence of the transient events. The increased average amplitude of the spectrum within the "noise notch" [Webb et al., 1991; Webb and Crawford, 2010] that is normally present in the power spectral densities of the seafloor pressure variations between the infragravity wave band and the microseism band was used as the screening criterion (Figure 2). Five percent of the recordings with the highest level of spectral harmonics at frequencies $50-60 \mathrm{mHz}$ were excluded from our analysis. The resulting power spectrum densities $S_{\mathrm{p}}$ of the seafloor pressure variations in the IGW frequency band are shown in Figure 3 for 28 MOANA stations. The spectra represent an average over the entire yearlong observation record.

[10] The power spectrum densities $S_{\mathrm{p}}$ obtained at stations with different water depths $H$ become rather similar when viewed as functions of the dimensionless argument $a=\omega^{2} H / g$, where $g$ is acceleration due to gravity. Within the accuracy of the measurements, the modified spectrum $\widetilde{S}_{\mathrm{p}}=\omega^{4} S_{\mathrm{p}}(\omega)$ has a maximum at the same value of $a \approx 1.1$ for all the stations (Figure $3 \mathrm{~b}$ ). The same result is obtained when a different procedure [Yang et al., 2012], which employs probability density functions of spectral density estimates instead of data screening, is applied to obtain $S_{\mathrm{p}}$ from the DPG data. Similar observations that maxima of IGW spectral density occur at the same $a$ for different $H$, recently have been made for the power spectra of the vertical component of the seafloor acceleration measured

$$
F(\omega)=\frac{6.459234 \cdot 10^{6} \omega^{2}(-i \omega+11656.0) \quad \text { counts } / \mathrm{Pa}}{(-i \omega+0.01667)(-i \omega+57.4710)(-i \omega+100.0)(-i \omega+0.2128)(-i \omega+1655.6)},
$$

where $\omega$ is circular frequency in $\mathrm{rad} / \mathrm{s}$. The variations in the laboratory-measured sensitivities and corner frequencies of 10 DPGs, of identical design to those used in the MOANA array, were found to be $10 \%$ and $25 \%$, respectively. The $25 \%$ includes one outlier. With the outlier excluded, the variation of the corner frequencies was $10 \%$.

[8] Tidal signals are dominant in seafloor pressure variations, and preventing aliasing of tidal energy is crucial in estimating wave spectra. IGW spectra were obtained by averaging the power spectral densities calculated for multiple data segments with the length equal to the period $T_{M 2} \approx 12.42$ $\mathrm{h}$ of the dominant tidal component, lunar tide M2. The Hann window was applied to the data segments to suppress any influence of other tidal components. As confirmed by computer simulations, the combination of these two simple steps reduced the contributions of parasitic harmonics of the tides by $\sim 15$ orders of magnitude in the IGW frequency band, thus making more sophisticated, model-based detiding procedures unnecessary for our purposes. by the 29-sensor OBS array in the MOANA experiment [Yang et al., 2012] (maxima are located at $a \approx 1.4$ ) and from smaller OBS arrays in the French Polynesia and the Philippine Sea [Sugioka et al., 2010] (maxima at $a \approx 2.0$ ).

[11] The least-squares data fit (Figure 3c) shows that dependence of the maximum of $\widetilde{S}_{\mathrm{p}}(a)$ on the ocean depth $H$ is well approximated by the power law $H^{-2}$.

\section{Theory}

[12] Because of their large free path length and a wide spatial distribution of the wave sources, background IGWs in deep water form a diffuse (but not necessarily isotropic) wave field. With a large number of incoherent wave sources contributing to IGW intensity either concurrently or over the duration of the observations, one may assume that the effects on the intensity of propagation paths between the sensor and individual wave sources average out. Then, to a first approximation, IGW spectra throughout an ocean basin can be 

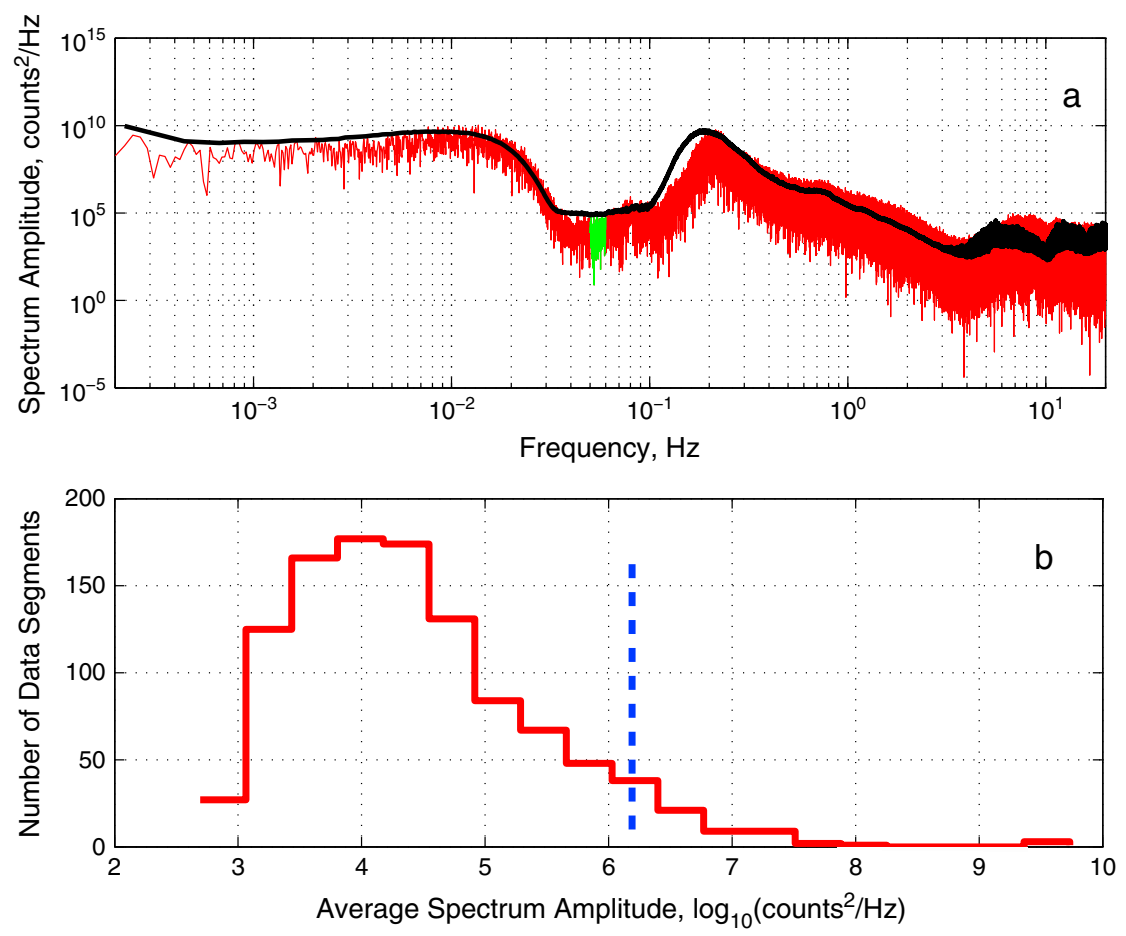

Figure 2. Screening of DPG data for transient events. (a) The initial step in data evaluation. Power spectral density calculated for a single data segment is shown by the red line, the average spectrum is shown by the black line, and the frequency band $50-60 \mathrm{mHz}$, which is used in the screening criterion, is marked in green. Data screening precedes the translation of the DPG instrument counts into the measured pressure, and the spectral densities are calculated in units of counts $^{2} / \mathrm{Hz}$. (b) Statistical distribution of the spectral amplitudes within the $50-60 \mathrm{mHz}$ range. The vertical dashed line marks the $5 \%$ percentile boundary in the $50-60 \mathrm{mHz}$ range.

represented as a product of a global characteristic of the wave sources (source spectrum) and a local characteristic of the wave, e.g., IGW phase speed. It follows from the first principles that such a representation is exact, rather than approximate, for perfectly diffuse wave fields such as thermal noise, see, e.g., [Godin, 2009]. An interferometric study [Zabotin et al., 2012] confirms that IGWs observed in the MOANA experiment are well described as a random diffuse wave field.

[13] Phase speeds of IGWs far exceed velocities of currents in the ocean, and ocean depth is much larger than the amplitudes of tides. Neglecting the effects of tides and currents, one has a stationary propagation environment for IGWs. Thus, the frequency $\omega$ of a continuous IGW does not change during propagation, while the IGW's wave number $k$ changes when the wave propagates in a horizontally inhomogeneous ocean. With wave frequency being a global characteristic, random wave sources (i.e., IGW forcing) can be characterized by a function $F\left(\omega / \omega_{0}\right)$, where $\omega_{0}$ is an arbitrarily chosen normalization frequency. Note that $F\left(\omega / \omega_{0}\right)$ will be a common factor in spectra of different observables (seafloor pressure, seafloor acceleration, ocean surface elevation, etc.) characterizing the IGW field in a given area.

[14] In considering IGWs in the ocean, one can treat the water as an incompressible fluid. Corrections due to water compressibility can be shown to be of the order of the square of the ratio of the IGW phase velocity to the sound speed, and are typically less than $0.5 \%$. Seafloor slope is typically much smaller than unity, and therefore does not affect, to first order, the local propagation conditions as expressed by the IGW dispersion relation. In this study, we are interested in IGWs with periods less than $2000 \mathrm{~s}$. The frequencies of such waves are much higher than the Coriolis parameter, which describes the effects of the Earth's rotation. The effect of the Earth rotation on the IGW dispersion relation can be shown to be of the second order in the ratio of the Coriolis parameter to the wave frequency, and is therefore negligible, except for certain coastally trapped waves such as Kelvin waves [Gill, 1982; Godin, 2012]. We consider points located sufficiently far from the coastline so that contributions of coastally trapped waves into the IGW field are insignificant. The effect of the atmosphere on propagation of IGWs in the ocean is negligible because water density exceeds air density by almost three orders of magnitude. Variations of water density $\rho$ with depth are small (a few percent) but crucial for internal gravity waves [Gill, 1982]. For IGWs, the density variations within the water column can be neglected, to first order, compared to the density jump on the sea surface. Having made the standard assumption that seafloor compliance is negligible [Webb et al., 1991; Uchiyama and McWilliams, 2008], one is then left with the only local parameter, the water depth $H$, to characterize the local propagation conditions.

[15] Consider the power spectrum $S_{\mathrm{p}}$ of IGW pressure on the seafloor. Pressure $p$ has a dimensionality of $\rho g^{2} \omega^{-2}$, where $g$ is the acceleration due to gravity. $S_{\mathrm{p}}$ has a dimensionality of $p^{2} / \omega$. There exists only one dimensionless combination, $a=\omega^{2} H / g$, of the dimensional parameters $g, \rho$, $H$, and $\omega$, that characterize IGWs at a given location. Hence, 
GODIN ET AL.: INFRAGRAVITY WAVES IN A DEEP OCEAN
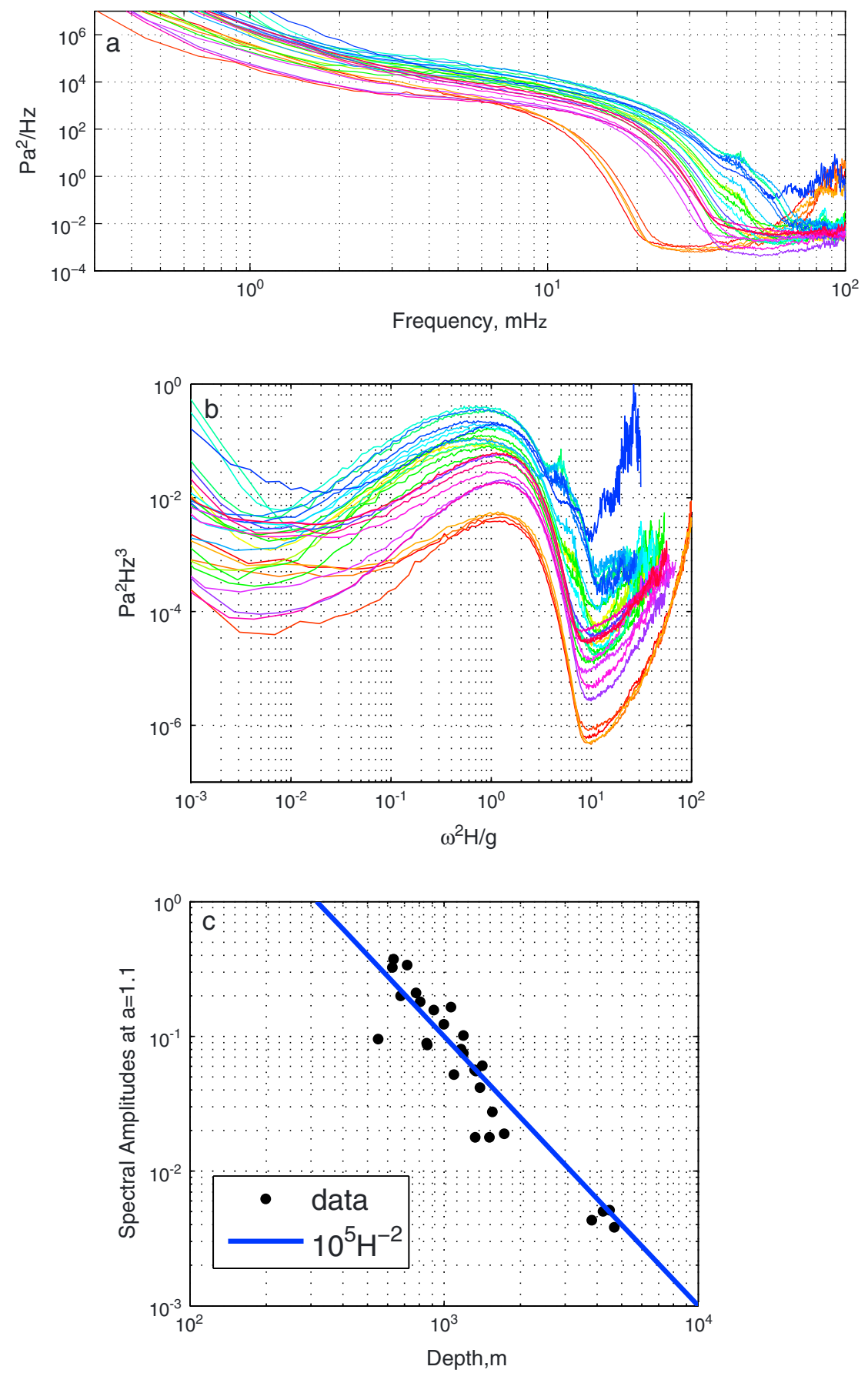

Figure 3. Measured spectra of pressure variations on the seafloor. (a) Average power spectra $S_{\mathrm{p}}$ of background pressure variations measured by 28 differential pressure gauges are shown as functions of frequency. (b) Modified power spectra $\widetilde{S}_{\mathrm{p}}=\omega^{4} S_{\mathrm{p}}$ are shown as functions of the dimensionless parameter $a=\omega^{2} \mathrm{H} / \mathrm{g}$. (c) Dependence of the measured spectral amplitudes at $a=1.1$ on water depth $H$ (dots) can be approximated by a power law (straight line). The dots in Figure $3 \mathrm{c}$ are the values at $a=1.1$ of the corresponding spectra shown in Figure $3 \mathrm{~b}$.

$$
S_{\mathrm{p}}(\omega, H)=g^{4} \rho^{2} \omega^{-5} F\left(\omega / \omega_{0}\right) f(a), \quad a=\omega^{2} H / g,
$$

where $\omega_{0}>0$ has the dimensionality of the frequency and is otherwise arbitrary; $F$ and $f$ are unknown nonnegative, dimensionless functions. These functions will be determined from the experimental data (Figure 3).

[16] Because the observed spectrum $\widetilde{S}_{\mathrm{p}}=\omega^{4} S_{\mathrm{p}}$ has a maximum at the same value $a_{0}$ of $a$ for different $H$, the partial derivative

$$
\left(\frac{\partial}{\partial \omega} \ln \widetilde{S}_{\mathrm{p}}(\omega, H)\right)_{H}=\frac{1}{\omega}\left[\frac{\omega^{2}}{F} \frac{\partial}{\partial \omega}\left(\frac{F}{\omega}\right)+2 a \frac{f^{\prime}}{f}\right]
$$

equals zero at $a=a_{0}$ for various $\omega$. Here prime denotes a derivative of a function with respect to its argument. Note that the first term in the square brackets in the right-hand side of equation (2) is a function of $\omega$, while the second term is a function of $a$. Hence, 

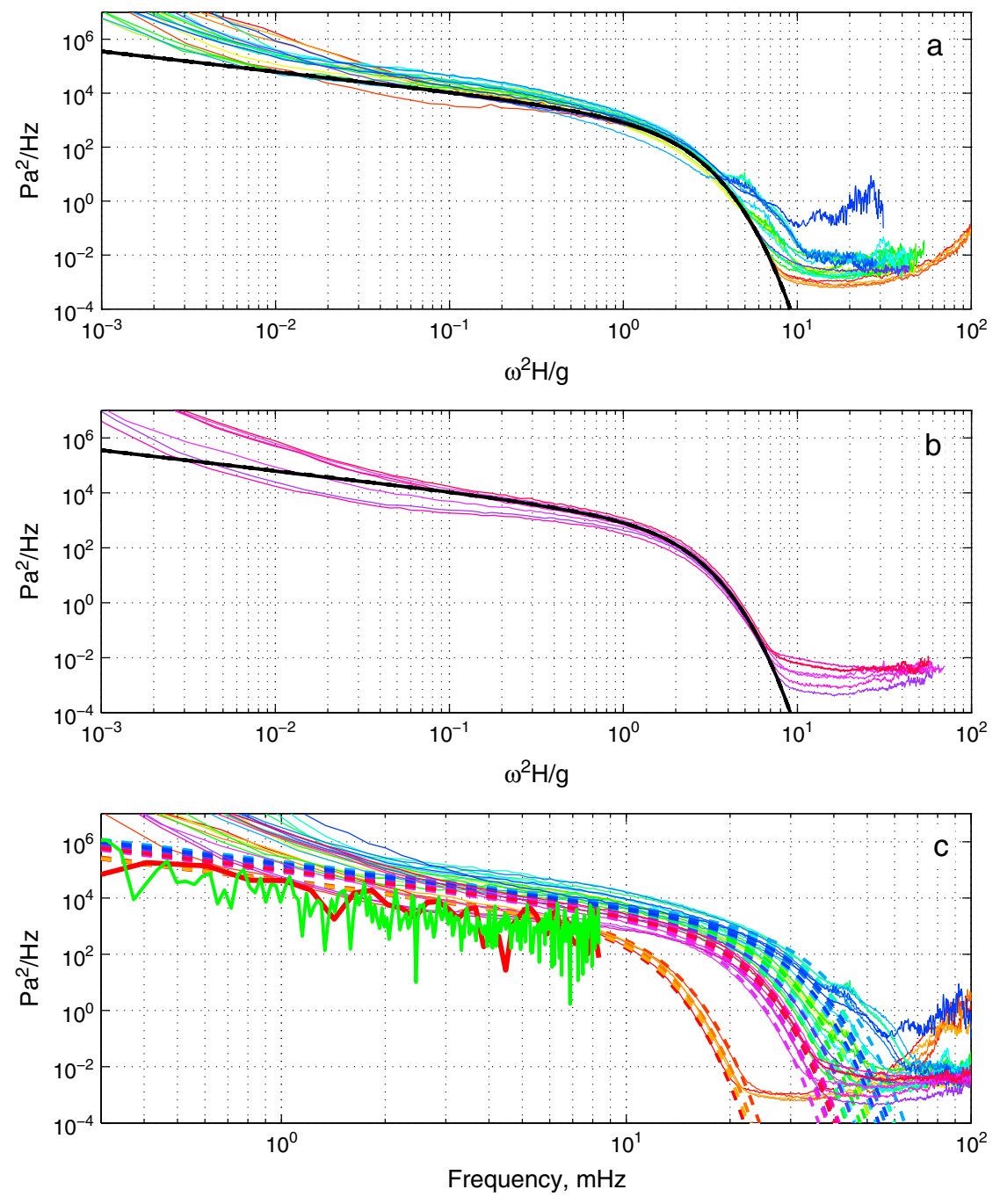

Figure 4. Comparison of the IGW spectral model to observations. The model of the power spectrum (heavy black line) is superimposed on results of DPG measurements (thin color lines) at (a) stations 1-21 to the northwest and at (b) stations 22-30 to the southeast from New Zealand's South Island. (c) The power spectra of the seafloor pressure measurements on DART\#55013 (heavy red and green lines) complement the power spectra of DPG measurements (thin, color-coded solid lines) and support the model predictions (dashed lines) at low frequencies. The power spectra are shown as functions of the dimensionless parameter $a=\omega^{2} H / g$ in Figures $4 \mathrm{a}$ and $4 \mathrm{~b}$ and as functions of the frequency in Figure $4 \mathrm{c}$.

$$
\frac{\omega^{2}}{F} \frac{\partial}{\partial \omega}\left(\frac{F}{\omega}\right)=-\beta
$$

where $\beta=2 a_{0} f^{\prime}\left(a_{0}\right) / f\left(a_{0}\right)$ is a constant. Solving the differential equation (3), we obtain $F\left(\omega / \omega_{0}\right)=$ const. $\cdot\left(\omega / \omega_{0}\right)^{1-\beta}$. Thus, IGW sources have a power-law spectrum.

[17] Using equation (1), we find that $\widetilde{S}_{\mathrm{p}} \sim H^{\beta / 2}$ at fixed $a$. Comparison with the experimental observation $\widetilde{S}_{\mathrm{p}} \sim H^{-2}$ gives $\beta=-4$ and, hence,

$$
S_{\mathrm{p}}=B g^{4} \rho^{2} \omega_{0}^{-5} f(a), \quad B=\text { const } .
$$

[18] Similar analysis of IGWs could be carried out using OBS data if the seafloor compliance were known a priori.

\section{Spectrum Model}

[19] Away from the shore, IGWs are free surface gravity waves with the dispersion relation $a=k H \tanh k H$, ocean surface displacement $h=p(x, y, 0) / \rho g$, and the dependence

$$
p(x, y, z)=p(x, y, 0) \cosh \mathrm{k}(H-z) / \cosh k H
$$

of the pressure on depth $z$ [Gill, 1982; Webb et al., 1991]. Here $x$ and $y$ are horizontal coordinates, $H=H(x, y)$ is the water depth; $z=0$ on the ocean surface and $z=H$ on the seafloor. Note that there is a one-to-one correspondence between $a \geq 0$ and the product $k H$ of the IGW wave number and the water depth.

[20] Equation (4) indicates that seafloor pressure spectra should collapse into a single curve when viewed as a function of either $a$ or $k H$. We have used observations at the stations 1-21 to derive a simple analytical model of the IGW spectra

$$
\begin{aligned}
S_{\mathrm{p}}(\omega, H) & =B_{p}(\tanh k H)^{-3 / 2} \cosh ^{-2} k H, \\
B_{\mathrm{p}} & =2.0 \cdot 10^{3} \mathrm{~Pa}^{2} / \mathrm{Hz} .
\end{aligned}
$$

[21] The spectrum model (6), which is obtained by fitting data obtained to the northwest of the South Island (Figure 4a), describes equally well the IGW spectra derived from observations at stations $22-30$ located to the southeast of the island (Figure 4b). 
[22] Within the IGW frequency band, the remaining scatter between the spectra measured at different stations (Figures $4 \mathrm{a}$ and $4 \mathrm{~b}$ ) can be attributed to differences between individual DPGs and the deviations of the respective transfer functions from the nominal transfer function we have used. Strong deviations from the model (6) occur at high frequencies (above $20-30 \mathrm{mHz}$, depending on ocean depth) (Figures $4 \mathrm{a}$ and $4 \mathrm{~b}$ ), where $k H$ is large and seafloor pressure due to IGWs is exponentially small according to equation (5). Flow noise and electronic noise dominate DPG records at such frequencies [Webb et al., 1991]. The model (6) also underestimates the measured spectra at the opposite extreme of low frequencies (below $\sim 1 \mathrm{mHz}$ ) (Figure 4), where DPGs are known to become unreliable [Cox et al., 1984; Webb et al., 1991]. DART measurements, which are of absolute rather than differential pressure, are free from low-frequency limitations, and have been used to evaluate the spectral model (6) at frequencies below $\sim 1 \mathrm{mHz}$.

[23] There was a single operational DART buoy, \#55013, in the vicinity of the MOANA experiment. In its standard mode, this DART buoy provides data with a $900 \mathrm{~s}$ sampling interval, which is too long for our purposes. Only when a tsunami alert is released, does this station provide data with a $60 \mathrm{~s}$ sampling interval. Two longest records of this kind ( $\sim 9 \mathrm{~h}$, starting on 19 March 2009 at 17:00 UTC and $\sim 1.5 \mathrm{~h}$, starting on 15 July 2009 at 8:00 UTC) have been de-tided (courtesy of Dr. G. Mungov, NOAA/NGDC) and used to calculate a spectrum of the seafloor pressure variations. The result (Figure 4c) indicates that the model (6) successfully describes low-frequency IGWs beyond the domain of applicability of the DPG measurements.

[24] For the spectrum of ocean surface elevations due to IGWs, from equations (5) and (6) we find $S_{\mathrm{h}}(\omega, H)=B_{\mathrm{h}}$ tanh ${ }^{-3 / 2} \mathrm{kH}$, where $B_{\mathrm{h}}=2.1 \cdot 10^{-5} \mathrm{~m}^{2} / \mathrm{Hz}$. Because IGW energy density per unit surface area is $\rho g h^{2}, S_{\mathrm{h}}$ describes the spectral distribution of the energy of the background IGWs. The spectrum $S_{\mathrm{h}}$ rapidly decreases with water depth as $H^{-3 / 2}$ at low frequencies $(a \ll 1)$ and becomes independent of $H$ at high frequencies $(a>2)$. The total IGW energy per unit surface area, $E$, which is obtained by integration of $\rho g S_{\mathrm{h}}$ in the frequency range $2 \pi f_{\min }<\omega<2 \pi f_{\max }$, is closely approximated by the contribution $E=\rho g^{7 / 4} B_{\mathrm{h}}$ $\left(2 \pi^{3} f_{\min }\right)^{-1 / 2} H^{-3 / 4}$ of the low-frequency IGWs. RMS surface displacement in IGWs, as predicted by the model (6) with $f_{\min }=0.3 \mathrm{mHz}$ and $f_{\max }=30 \mathrm{mHz}$, is shown in Figure 1 .

\section{Conclusion}

[25] The energy density of IGWs away from the shore strongly depends on water depth. Being combined with a theory of diffuse wave fields in inhomogeneous media, observations with an extensive network of seafloor pressure sensors off New Zealand led to a simple, analytical model (6) of the spectral and spatial distribution of IGW energy in a deep ocean. The model has far reaching implications for the theory of generation of deep-water IGWs and can be used to predict noise levels in future ocean-bottom seismic experiments, help design experiments on measurements of seafloor compliance, calibrate differential pressure gauges in situ, and quantify the interaction of IGWs with the atmosphere and ice shelves. Application of the technique developed in this paper to noise records obtained in past and future ocean-bottom seismic experiments will allow one to study the geographic variability of the background IGWs and to extend the spectrum model (6) to other regions of the ocean.

[26] Acknowledgments. The collection of DPG data was supported by the National Science Foundation Continental Dynamics program under grants EAR-0409564, EAR-0409609, and EAR-0409835. The instruments were provided by the U.S. National Ocean Bottom Seismic Instrumentation Pool (http://www.obsip.org). We thank G. Mungov for help with accessing and pre-processing of the DART data provided by NGDC, and J. Ball for input on DPG variability and calibration. Thoughtful comments by W. Crawford, B. Romanowicz and two anonymous reviewers are gratefully acknowledged. This work is supported by the University of Colorado Innovative Seed Grant (IGP) "Study of Ocean Infragravity Waves with a Large Array of Seafloor Seismometers."

[27] The Editor thanks Wayne Crawford, Barbara Romanowicz and anonymous reviewers for their assistance in evaluating this paper.

\section{References}

Bromirski, P. D., O. V. Sergienko, and D. R. MacAyeal (2010), Transoceanic infragravity waves impacting Antarctic ice shelves, Geophys. Res. Lett., 37, L02502, doi:10.1029/2009GL041488.

Bromirski, P. D., and R. A. Stephen (2012), Response of the Ross Ice Shelf, Antarctica, to ocean gravity-wave forcing, Ann. Glaciol., 53(60), 163-172.

Cox, C., T. Deaton, and S. Webb (1984), A deep-sea differential pressure gauge, J. Atmos. Oceanic Technol., 1, 237-246.

Crawford, W. C., S. C. Webb, and J. A. Hildebrand (1998), Estimating shear velocities in the oceanic crust from compliance measurements by two-dimensional finite difference modeling, J. Geophys. Res., 103(B5), 9895-9916.

Dolenc, D., B. Romanowicz, D. Stakes, P. McGill, and D. Neuhauser (2005), Observations of infragravity waves at the Monterey ocean bottom broadband station (MOBB), Geochem. Geophys. Geosyst., 6, Q09002, doi:10.1029/2005GC000988.

Dolenc, D., B. Romanowicz, P. McGill, and W. Wilcock (2008), Observations of infragravity waves at the ocean-bottom broadband seismic stations Endeavour (KEBB) and Explorer (KXBB), Geochem. Geophys. Geosyst., 9, Q05007, doi:10.1029/2008GC001942.

Filloux, J. H. (1983), Pressure fluctuations on the open ocean floor off the Gulf of California: Tides, earthquakes and tsunamis, J. Phys. Oceanogr., 13, 783-796.

Gill, A. E. (1982), Atmosphere-Ocean Dynamics, Academic Press, New York.

Godin, O. A. (2009), Retrieval of Green's functions of elastic waves from thermal fluctuations of fluid-solid systems, J. Acoust. Soc. Am., 125, 1960-1970.

Godin, O. A. (2012), Incompressible wave motion of compressible fluids, Phys. Rev. Lett., 108, 194501.

Godin, O. A., and I. M. Fuks (2012), Transmission of acoustic-gravity waves through gas-liquid interfaces, J. Fluid Mech., 709, 313-340.

Godin, O. A., and V. G. Irisov (2003), A perturbation model of radiometric manifestations of oceanic currents, Radio Sci., 38, 8070, doi:10.1029/ 2002RS002642.

Herbers, T. H. C., S. Elgar, and R. T. Guza (1995), Generation and propagation of infragravity waves, J. Geophys. Res., 100, 24863-24872.

Komen, G. J., L. Cavaleri, M. Donelan, K. Hasselmann, S. Hasselmann, and P. A. E. M. Janssen (1984), Dynamics and Modelling of Ocean Waves, Cambridge Univ, Press, Cambridge.

Munk, W. H. (1949), Surf beat, Eos Trans, AGU, 30, 849-854.

Rhie, J., and B. Romanowicz (2004), Excitation of Earth's continuous free oscillations by atmosphere-ocean-seafloor coupling, Nature, 431, $552-556$.

Sheremet, A., R. T. Guza, S. Elgar, and T. H. C. Herbers (2002), Observations of nearshore infragravity waves: Seaward and shoreward propagating components, J. Geophys. Res., 107 (C8), 3095.

Snodgrass, F. E., G. W. Groves, K. F. Hasselmann, G. R. Miller, W. H. Munk, and W. H. Powers (1966), Propagation of ocean swell across the Pacific, Phil. Trans. R. Soc. London, A259(1103), 431-497.

Sugioka, H., Y. Fukao, and T. Kanazawa (2010) Evidence for infragravity wave-tide resonance in deep oceans, Nat. Commun. 1, 84, doi:10.1038/ ncomms 1083 .

Uchiyama, Y., and J. C. McWilliams (2008), Infragravity waves in the deep ocean: Generation, propagation and seismic hum excitation, J. Geophys. Res., 113, C07029, doi:10.1029/2007JC004562. 


\section{GODIN ET AL.: INFRAGRAVITY WAVES IN A DEEP OCEAN}

Webb, S. C., X. Zhang, and W. Crawford (1991), Infragravity waves in the deep ocean, J. Geophys. Res., 96, 2723-2736.

Webb, S. C. (2007), The Earth's 'hum' is driven by ocean waves over the continental shelves, Nature, 445(7129), 754-756.

Webb, S. C., and W. Crawford (2010), Shallow-water broadband OBS seismology, Bull. Seismol. Soc. Am., 96, 2723-2736.

Willoughby, D. F., J. A. Orcutt, and D. Horwitt (1993), A microprocessor-based ocean-bottom seismometer, Bull. Seismol. Soc. Am., 83, 190-217.
Yang, Z., A. Sheehan, J. A. Collins, and G. Laske (2012), The character of seafloor ambient noise recorded offshore New Zealand: Results from the MOANA ocean bottom seismic experiment, Geochem. Geophys. Geosyst., 13, Q10011, doi:10.1029/2012GC004201.

Zabotin, N. A., O. A. Godin, A. Sheehan, Z. Yang, and J. A. Collins, 2012. Application of wave interferometry to experimental investigation of infragravity waves off New Zealand, 2012 Ocean Sci. Meeting, 20-24 February 2012, Salt Lake City, Utah. Paper 9322, http://www.sgmeet. com/osm2012/viewabstract2.asp?AbstractID $=9322$. 